\title{
Topological Insulator-to-Weyl Semimetal Transition in Strongly Correlated Actinide System UNiSn
}

\author{
Vsevolod Ivanov, ${ }^{1}$ Xiangang Wan, ${ }^{2}$ and Sergey Y. Savrasov ${ }^{1}$ \\ ${ }^{1}$ Department of Physics, University of California, Davis, California 95616, USA \\ ${ }^{2}$ National Laboratory of Solid State Microstructures, School of Physics and Collaborative Innovation \\ Center of Advanced Microstructures, Nanjing University, Nanjing, China
}

(Received 16 October 2018; revised manuscript received 28 October 2019; published 16 December 2019)

\begin{abstract}
Although strong electronic correlations are known to be responsible for some highly unusual behaviors of solids such as metal-insulator transitions, magnetism, and even high-temperature superconductivity, their interplay with recently discovered topological states of matter awaits a full exploration. Here, we use a modern electronic structure method, combining the density functional theory of band electrons with dynamical self-energies of strongly correlated states, to predict that two well-known phases of actinide compound UNiSn, a paramagnetic semiconducting and antiferromagnetic metallic, correspond to topological insulator (TI) and Weyl semimetal (WSM) phases of topological quantum matter. Thus, the famous unconventional insulator-metal transition observed in UNiSn is also a TI-to-WSM transition. Driven by a strong hybridization between $\mathrm{U} f$-electron multiplet transitions and band electrons, multiple energy gaps open up in the single-particle spectrum whose topological physics is revealed using the calculation of $Z_{2}$ invariants in the strongly correlated regime. A simplified physical picture of these phenomena is provided based on a periodic Anderson model of strong correlations and multiple band inversions that occur in this fascinating compound. Studying the topology of interacting electrons reveals interesting opportunities for finding exotic phase transitions in strongly correlated systems.

DOI: 10.1103/PhysRevX.9.041055

Subject Areas: Condensed Matter Physics,

Strongly Correlated Materials,

Topological Insulators
\end{abstract}

\section{INTRODUCTION}

Strongly correlated systems are known for a whole range of spectacular phenomena such as, e.g., colossal magnetoresistance of manganese oxides [1], high-temperature superconductivity of cuprates [2] and iron arsenides [3], enormous volume expansions in elemental cerium [4] and plutonium [5], heavy electron-mass renormalizations in compounds containing $f$ and, sometimes, $d$ electrons [6], etc. Recently, the theme of strong correlations has come into play with the notion of topology in electronic band structures, whose robust quantum states are insensitive to perturbations and are currently attracting a great interest in materials such as topological insulators (TIs) [7] and Weyl semimetals (WSMs) [8]. Starting from an original proposal that pyrochlore iridates, subjected to a moderate Coulomb repulsion of their $5 d$ electrons, should exhibit a dispersion in the vicinity of the bulk Fermi level characteristic of Weyl

Published by the American Physical Society under the terms of the Creative Commons Attribution 4.0 International license. Further distribution of this work must maintain attribution to the author(s) and the published article's title, journal citation, and DOI. fermions in particle physics and the associated Fermi-arc surface states [9], the field has been enriched by the discoveries of topological Kondo insulator [10] behavior in $\mathrm{SmB}_{6}$ [11] and filled skutterudites [12] and plutonium and americium TIs based on rocksalt structure [13], as well as heavy-fermion Weyl-Kondo semimetals [14]. These systems, representing a merge between paradigms of correlations and topology, could serve as the basis for studying yet-unknown electronic phases, transitions, and functionalities and may lead to interesting applications in the future.

Unfortunately, identifying topological properties of correlated electrons represents a challenge due to a wellknown problem associated with redistribution of spectral weight driven by the interplay between Coulomb repulsion and kinetic degrees of freedom for the electrons. As a result, signatures of localized electronic states originating from atomic multiplet transitions, known as Hubbard bands, as well as strongly renormalized quasiparticle bands in the vicinity of the Fermi level, often both appear in materials with strong correlations. Such competition between localization and delocalization is at the heart of the Mott transition problem [15], which has been well understood through the development of the dynamical meanfield theory (DMFT) [16]. This approach defies static 
mean-field approximation as, e.g., implemented in density functional theory (DFT) -based electronic structure calculations [17]. These methods provide a single-particle framework for computing topological indices [18-21] and are currently playing a central role in identifying topological materials, using, in particular, a powerful method of high-throughput screening that allows testing hundreds or even thousands of compounds [22-25]. The strongly correlated problem generally requires studying the topological character, such as Berry phases, of many-body wave functions $[19,26,27]$ and is primarily addressed on the level of many-body model Hamiltonians [28-30].

Nowadays, modern electronic structure approaches based on combinations of the local density approximation (LDA) [17] and DMFT (LDA + DMFT method) [31] allow for a more accurate treatment of Coulomb correlations via computations of local self-energies $\Sigma(\omega)$ for the interacting electrons. This computation is achieved by treating a correlated atomic shell as an impurity hybridized with the noninteracting bath, which is then periodized and subjected to self-consistency. Searches of correlated topological matter with the use of LDA + DMFT are advantageous, as they incorporate the detailed chemistry and structure of a material into the calculation. It is the purpose of this work to demonstrate how the LDA + DMFT method can be applied to uncover the rich topological behavior of the actinide system UNiSn and provide a framework for studying other compounds where genuine many-body effects need to be taken into account while searching for their topological properties.

An unusual phase transition at $T_{N}=43 \mathrm{~K}$ between a higher-temperature paramagnetic semiconducting (PMS) and low-temperature antiferromagnetic metallic (AFMM) phase was discovered for UNiSn long ago [32]. This actinide compound was extensively studied during the past several decades owing to the unconventional (inverse) nature of this metal-insulator transition with the gap opening above $T_{N}$ and the associated behavior of its strongly correlated $5 f$ electrons. It crystallizes in a cubic structure (MgAgAs type) [see Fig. 1(a)], and its paramagnetic semiconducting phase has an estimated energy gap of about $100 \mathrm{meV}$ [33]. Its antiferromagnetic structure is found to be of type I with the ordered $\mathrm{U}$ moment $1.55 \mu_{B}$ oriented along the (001) axis [32].

The central issue in understanding the physical properties of actinides is the degree to which their $5 f$ electrons are localized. Because of the absence of any signatures of heavy fermion behavior in the specific heat data [33], the magnetic properties of UNiSn are explained [34] on the basis of a localized $5 f^{2}\left(\mathrm{U}^{4+}\right)$ ionic state, whose ground state multiplet ${ }^{3} \mathrm{H}_{4}(J=4)$ subjected to a cubic crystal field is split into a doublet $\left(\Gamma_{3}\right)$, two triplets $\left(\Gamma_{4}\right.$ and $\left.\Gamma_{5}\right)$, and a singlet $\left(\Gamma_{1}\right)$ [35]. Measured temperature-dependent susceptibility and magnetic entropy analysis suggests that the nonmagnetic doublet is the lowest-lying state $180 \mathrm{~K}$ below

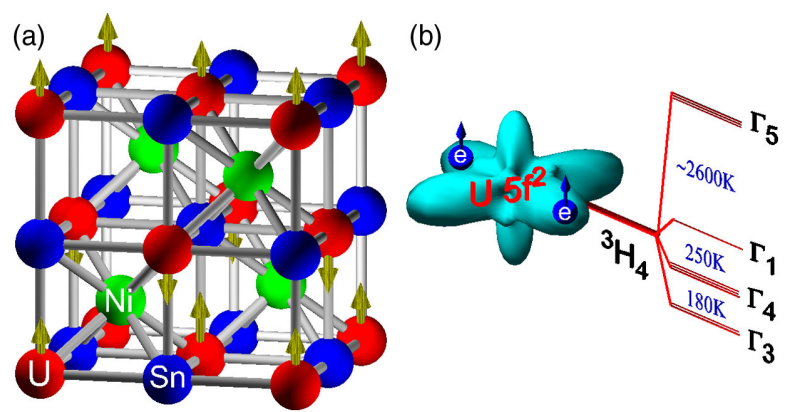

FIG. 1. (a) Crystal structure of UNiSn showing antiferromagnetic type-I ordering [32]. (b) Effect of the cubic crystal field splitting on the ${ }^{3} \mathrm{H}_{4}$ ground state multiplet of the $\mathrm{U} f^{2}$ twoelectron state with its lowest nonmagnetic $\Gamma_{3}$ doublet as found experimentally [34].

the $\Gamma_{4}$ triplet and $430 \mathrm{~K}$ below the $\Gamma_{1}$ singlet [see Fig. 1(b)]. Since $\Gamma_{3}$ has a quadrupole moment, it is further proposed that tetragonal distortions and quadrupolar ordering exists below $T_{N}$ [32]. The valence band photoemission spectra reveal a dominant $5 f$ electron character for the states in the vicinity of the Fermi level with a contribution from $\mathrm{U} 6 d$, Ni $3 d$, and Sn $6 p$ states [36].

Previous band structure calculations of UNiSn emphasize the role of relativistic effects and electronic correlations among $5 f$ electrons [37]. Both PMS and AFMM behavior are captured correctly within the LDA $+U$ framework [38], where on-site Coulomb correlations among $f$ electrons are treated via the introduction of the Hubbard $U$ term and subsequent static mean-field approximation. Such a method is expected to work well in a symmetry-broken AFM state but is invalid for the genuine two-electron $\Gamma_{3}$ doublet represented by a mixture of Slater determinants. One can, however, assume that paramagnetism originates from the nonmagnetic $\Gamma_{1}$ singlet, for which LDA $+U$ should be sufficient. Within a single-particle picture, this assumption is interpreted as a doubly occupied $\Gamma_{7}$ level that appears when a 14 -fold degenerate manifold of $5 f$ electrons subjected to spin-orbit coupling and a cubic crystal field is split into $\Gamma_{7}$ and $\Gamma_{8}$ (for $j=5 / 2$ ) and $\Gamma_{6}, \Gamma_{7}$, and $\Gamma_{8}$ (for $j=7 / 2$ ) sublevels. Detailed comparisons between the theory and experiment reveal discrepancies in the position of the occupied $f$ band with respect to the Fermi energy: $-0.3 \mathrm{eV}$ in the photoemission vs $-1 \mathrm{eV}$ in the LDA $+U$ calculation [36].

In the present work, we go beyond static mean-field approaches and utilize a modern LDA + DMFT method in order to account for the interaction effect among $5 f$ electrons more accurately. As our main result, we uncover that the two well-known phases of actinide compound UNiSn, correspond to TI and WSM phases of topological quantum matter. Thus, the unconventional insulator-metal transition observed in UNiSn is also a TI-to-WSM transition. The ability to trigger changes in topological phases by varying the temperature is interesting both 
fundamentally and from the point of view of applications, since metal-insulator transitions are very attractive, in general, for making all kinds of electrical and thermal conductivity switches or optical modulators. Using the magic of a highly conductive coating in terms of disordertolerant surface states that exist in TIs and WSMs can provide additional control in those applications, as, for example, Weyl semimetals thin films or nanostructures exhibiting ultrahigh conductivities $[39,40]$. Other functionalities could take advantage of very different responses to an applied magnetic field relevant for magnetotransport or differences between spin textures of the Dirac-cone or Fermi-arc states that could allow a temperature control of current-induced surface spin polarization relevant for spintronics $[41,42]$.

This paper is organized as follows: In Sec. II, we describe the details of how the LDA + DMFT method treats the correlated uranium $5 f$ electrons in this system. Section III is split onto two parts: In Sec. III A, we discuss the results of our calculations for paramagnetic insulating phase and prove its topological insulating behavior by computing both $Z_{2}$ invariants in the strongly correlated regime as well as its surface spectrum exhibiting the Diraccone states. A simplified physical picture based on the periodic Anderson model of strong correlations and multiple band inversions that occur in UNiSn is provided. In Sec. III B, we show the results of our calculation for the antiferromagnetic metallic phase and prove the existence of Weyl points in the vicinity of the Fermi level using our recently developed monopole mining method [25]. A $4 \times 4 \mathbf{k} \cdot \mathbf{p}$ model is developed here in order to illustrate the appearance of the Weyl points along the magnetization direction and the associated Fermi-arc surface states. In Sec. IV, we conclude with a perspective on other correlated uranium compounds that could exhibit related phenomena. An Appendix provides the details of our LDA + DMFT framework and the computation of topological invariants that is utilized in this work.

\section{METHOD}

An improved treatment of Coulomb interactions in UNiSn should include a frequency-dependent self-energy $\Sigma_{f}(\omega)$ within the spin-orbit coupled space of the $5 f$ electrons (dimension 14) which is incorporated into the single-particle LDA Hamiltonian describing all other (weakly correlated) states. A family of approaches that allow such a combination of the self-energy with LDA (the SELDA family) has been developed with the help of projector operators long ago [43]. The LDA + DMFT method [31] delivers $\Sigma_{f}(\omega)$ by iteratively solving an auxiliary Anderson impurity model (AIM) that considers hybridization between $5 f$ states and other noninteracting electrons as a self-consistent function that changes during the DMFT iterations. The charge density self-consistency is subsequently utilized in a manner prescribed by the DFT.
The exact solution of the AIM is possible, in principle, via a recently developed continuous time quantum Monte Carlo (CTQMC) method [44], although accounting for the full Hilbert space of interacting $f$ electrons together with spinorbit and crystal field terms represents a challenge. In addition, the CTQMC works on the imaginary timefrequency axis, and obtaining the frequency dependence of the self-energy on the real axis involves an analytical continuation algorithm, which is known to be not very accurate.

In order to study the topology of correlated electrons in UNiSn here, we take a pragmatic approach and make the DMFT impurity problem numerically tractable by using the experimental fact that the uranium $f$ electrons are localized in their $5 f^{2} \Gamma_{3}$ ground state, from which the one-electron multiplet transitions can be obtained by exact diagonalization. The corresponding $f$-electron self-energies are subsequently expanded in the Laurent series, which allows us to replace the nonlinear (in energy) Dyson equation by a linear Schrödinger-like equation in an extended subset of "pole states" [45] (see the Appendix for details). Remarkably, the pole representation for the self-energy results in the appearance of many-body satellites and multiplets in the spectra as effective band states, in general, carrying a fractional occupancy due to the spectral weight transfer. It is ideally suited for studying topological indices, as the corresponding auxiliary wave functions representing the many-body features carry all the necessary information about the Berry phase of the interacting electrons [46].

For UNiSn, we carry out all calculations by treating the $f$ electrons in their $5 f^{2} \Gamma_{3}$ ground state. The Coulomb interaction matrix elements needed for the exact diagonalization procedure $\left(F^{(0)}, F^{(2)}, F^{(4)}\right.$, and $F^{(6)}$ Slater integrals) are found from the atomic $5 f$-electron wave functions and scaled to account for screening effects. We cover a range of these parameters: $2-4 \mathrm{eV}$ for the Hubbard $U=F^{(0)}$ and $0-1 \mathrm{eV}$ for the exchange $J=\left(286 F^{(2)}+195 F^{(4)}+250 F^{(6)}\right) /$ 6435 , in order to make sure that our conclusions are not altered by the lack of an accurate procedure for determining the screening. It has been argued earlier that these values are typical for obtaining the best agreement between the theory and experiment for several uranium compounds $[36,37]$. The position of the bare $f$ level is fixed by reproducing the experimentally observed $f^{2} \rightarrow f^{1}$ electron removal transition at $-0.3 \mathrm{eV}$ [36]. The charge density self-consistency is carried out within LDA + DMFT as implemented by one us earlier [47]. For a PM calculation, the spin-up and spin-down self-energies are forced to be equivalent, which prevents developing a magnetic state. The AFM instability is studied by first introducing a staggered magnetic field perturbation and letting the self-consistent solution converge. If sustainable, the spinup and spin-down self-energies become different, and the solution develops magnetic moments on corresponding sites of the lattice. 


\section{RESULTS}

\section{A. Paramagnetic phase}

We now present the results of our calculation for the paramagnetic phase of UNiSn. Figure 2 shows our calculated many-body electronic spectrum in the vicinity of the Fermi level using a set of Slater integrals $F^{(0)}=0.15$, $F^{(2)}=0.3, F^{(4)}=0.2$, and $F^{(6)}=0.15$ in Rydberg units. Although cast into a conventional band structure plot, we stress that the $5 f$ electron states are treated here as true oneelectron removal $\left(f^{2} \rightarrow f^{1}\right)$ and addition $\left(f^{2} \rightarrow f^{3}\right)$ processes that come from exact diagonalization, and the corresponding "energy bands" carry noninteger occupation. This treatment can be seen by realizing that the multiplet transitions within the $j=5 / 2$ manifold (shown in Fig. 2 by red and blue) are represented by six energy bands that appear both below and above the Fermi level. These are the famous lower and upper Hubbard bands within the Mott gap picture that acquire a significant dispersion due to hybridization with $\mathrm{U} 6 d$ and Sn $5 p$ orbitals. The deduced value of the indirect energy gap shows some dependence on the Slater integrals but falls into the same range as the experiment ( 100 meV [33]).

We now turn to the prediction of topological properties for the paramagnetic semiconducting phase of UNiSn. First, we point out that the underlying crystal structure

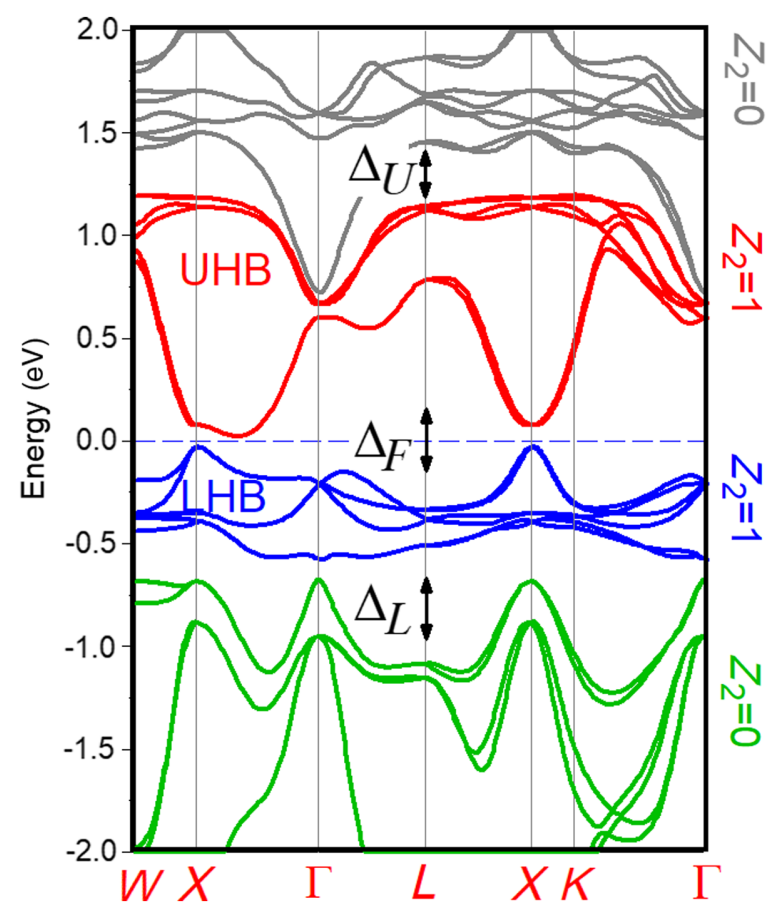

FIG. 2. Calculated electronic structure of UNiSn using the density functional theory combined with dynamical self-energies for the uranium $f$ electrons assuming the experimentally determined $5 f^{2} \Gamma_{3}$ doublet as a ground state [34]. The locations of energy panels with nonzero $Z_{2}$ invariants and corresponding gaps $\left(\Delta_{L}, \Delta_{F}\right.$, and $\left.\Delta_{U}\right)$ are indicated. is not centrosymmetric; therefore, the $\mathrm{Fu}$ and Kane parity criterion [18] developed for insulators with both time reversal and inversion symmetries does not apply. Nevertheless, given the fact that the uranium sites arrange themselves on an inversion symmetric face-centered cubic sublattice with their odd-parity localized $5 f$ electrons lying in close proximity to the Fermi level, it is interesting to speculate whether the possibility of inversion with the evenparity $\mathrm{U} 6 d$ band is taking place. Such an $f-d$ band inversion is at the center of recent interest for several topological Kondo insulator materials with $4 f$ electrons [10], as well as in some actinide systems such as AmC [13]. While the $\mathrm{U} 6 d$ band is expected to be unoccupied, it is very wide, with its lower portion hybridized with the Hubbard bands. The Fu and Kane criterion then implies the existence of topological Dirac-cone states in UNiSn.

To uncover the topological physics, one needs to compute $Z_{2}$ invariants [19] for the occupied band manifold in the difficult regime of strong correlations. Fortunately, it was recently proved that utilizing a pole representation for the self-energy [45] reduces this problem to an effective noninteracting system in the extended set of pole states, whose topological indices are exactly matched [46]. We develop and carry out this computation within the $n$-field approach [21] (see the Appendix for details). However, some care should be taken to define an appropriate energy panel, because, as is seen from our calculations, multiple gaps appear in the excitational spectrum of UNiSn (we show the panels by various colors and denote the gaps between them as $\Delta_{L}, \Delta_{F}$, and $\Delta_{U}$ in Fig. 2). For example, the six dispersive features that represent the lower Hubbard bands (blue "spaghetti" in Fig. 2 labeled as LHB) are completely gapped from the remaining band manifold everywhere in the BZ. The same is seen for the six eigenstates representing the upper Hubbard bands above the $E_{F}$ (red spaghetti in Fig. 2 labeled UHB). Our computations of $Z_{2}$ invariants for the four energy panels separated by $\Delta_{L}, \Delta_{F}$, and $\Delta_{U}$ reveal their topological indices, which we indicate in the right margin in Fig. 2. The energy panels below and above the fundamental gap correspond to the indices equal to $1 ;(000)$ in the notations of Ref. [19] (we denote this result by $Z_{2}=1$ in Fig. 2). This computation proves that $\mathrm{UNiSn}$ is a strong topological insulator and suggests the existence of protected Diraccone states at its surface.

To understand which orbitals are responsible for the appearance of the topological phase, we carry out calculations using a constrained hybridization approach [48]. In this method, the energies of particular orbitals are shifted by applying a constant potential constrained within the orbital space by projector operators. This method is similar to the $\mathrm{LDA}+U, \mathrm{LDA}+\mathrm{DMFT}$, and other SELDA families of methods, restricting the application of the self-energy to the subspace of correlated orbitals. Utilizing this procedure, we are able to dehybridize various states, such as $\mathrm{U}-5 f$, 

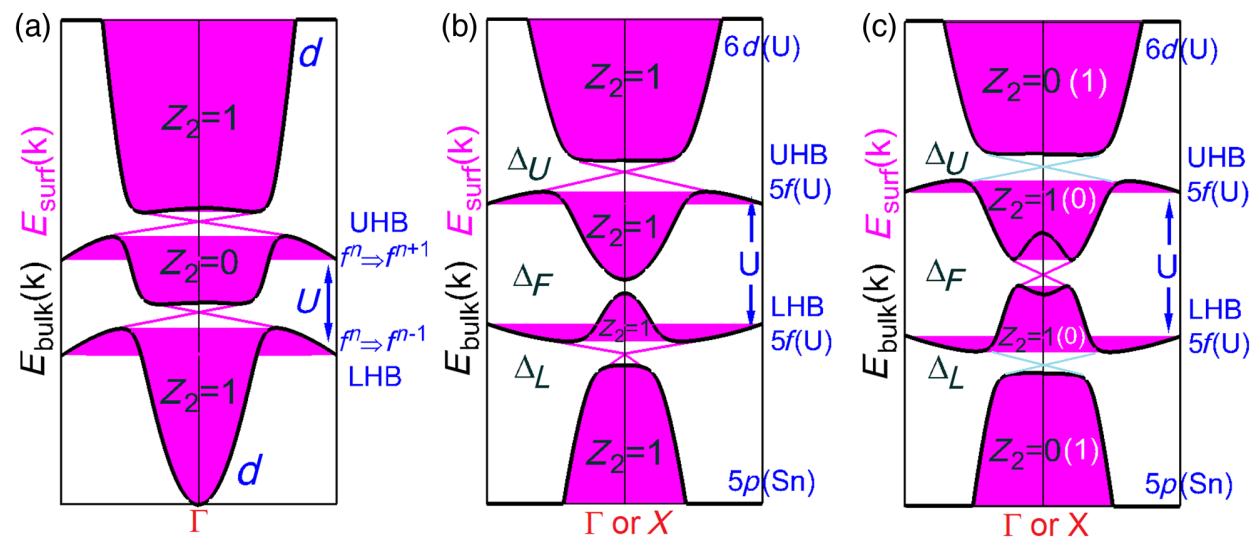

FIG. 3. Band inversion mechanism applicable for UNiSn. (a) In the periodic Anderson model, hybridization between a wide $d$ band centered at the $\mathbf{k}=0 \Gamma$ point and $f$-electron multiplet transitions [lower and upper Hubbard bands (LHB and UHB, respectively)] results in three energy panels (shown by black lines) and two gaps that are both topologically nontrivial. The corresponding surface spectrum is shown by magenta, where the spectral weight of the Dirac cone is distributed between the two gaps. (b) In UNiSn, the upper Hubbard band is inverted with the wide $6 d$ band of uranium, while the lower Hubbard band is inverted with the $5 p$ band of tin, resulting in four energy panels and three gaps. With two such band inversions, upper $\left(\Delta_{U}\right)$ and lower $\left(\Delta_{L}\right)$, the hybridization gaps are topological, while the fundamental bulk gap $\Delta_{F}$ is not. (c) The band inversion between $\mathrm{U} 6 d$ and $\mathrm{Sn} 5 p$ states around the zone boundary $X$ point makes the fundamental gap $\Delta_{F}$ topological. The topological features of the gaps $\Delta_{U}$ and $\Delta_{L}$ are seen to disappear in the LDA + DMFT calculation, but this disappearance is not a requirement within the considered model $\left(Z_{2}\right.$ invariants shown in parentheses are expected).

$\mathrm{U}-6 d$, Ni-3d, Sn-5p, etc., by shifting their energies away from the relevant energy window, and recompute $\mathrm{Z}_{2}$ invariants. The outcome of this study is the existence of multiple band inversions in UNiSn: The upper Hubbard band is inverted with the $\mathrm{U} 6 d$ electrons around the $\Gamma$ point of the BZ, while the lower Hubbard band is inverted with $\mathrm{Sn} 5 p$ electrons. On top of that, $\mathrm{U} 6 d$ electrons at the very bottom of the conduction band and $S n 5 p$ states at the very top of the valence band are also inverted around the $X$ point of the BZ (see Fig. 2). These multiple band inversions are responsible for the topological insulator behavior in UNiSn.

To illustrate the emergent physical picture, we use the periodic Anderson model (PAM) of strong correlations. It has been recently employed for developing the concept of topological Kondo insulators where the Fermi level falls into the gap between a heavy fermion ( $f$-like) and noninteracting ( $d$-like) bands [10]. It has also been recently used to describe Weyl-Kondo semimetals via hybridization of a heavy-fermion state with noninteracting bands containing the nodal points [14]. In our case, the $f$ electrons are localized, and their self-energies behave similarly to the famous Hubbard I approximation: $\Sigma(\omega)=U^{2} / 4 \omega$. The solution of the PAM in this limit is schematically illustrated in Fig. 3(a). Hybridization between wide $d$-band and $f$-electron multiplet transitions denoted as LHB and UHB results in the appearance of two gaps in the spectrum and three energy panels (shown by black lines). Both gaps are seen to be topologically nontrivial due to the $d-f$ band inversion mechanism. For a centrosymmetric lattice, this mechanism can be understood based on the $\mathrm{Fu}$ and Kane parity criterion [18]: For the lower (upper) panel, the parities of the eigenstates are odd (even) at the $X$ and $L$ time reversal invariant momenta (TRIM) but even (odd) at $\Gamma$. As a result, the energy gap above (below) the panel is topological. For the central panel, the parities of the eigenstates are odd everywhere, but this result does not preclude having a topological gap both below and above the panel, each with its own Dirac cone (the total number of cones is even). We illustrate the corresponding surface spectrum in magenta. Note that, since the Hubbard bands carry no integer occupation, the spectral weight of the Dirac cones is also redistributed between the two gaps.

Now, in UNiSn, our constrained hybridization procedure reveals multiple band inversions around different TRIM points in the BZ: First, as illustrated in Fig. 3(b), the upper Hubbard band is inverted with the $\mathrm{U} 6 d$ at $\Gamma$. Because of the $d-f$ band inversion, the topological Dirac cone is expected to appear inside the gap $\Delta_{U}$ at the surface spectrum. Since uranium atoms occupy sites of the centrosymmetric face-centered cubic lattice, this expectation can be understood based on the parity criterion [18]. For the lower Hubbard band, U $5 f$ and Sn $5 p$ orbitals are both odd but belong to different irreducible representations, which makes it possible to produce a strong topological insulator with a Dirac cone inside the gap $\Delta_{L}$. This picture emerges when the bottom of the $\mathrm{U} 6 d$ and the top of the Sn $5 p$ bands are not inverted around the $X$ point, making the fundamental gap $\Delta_{F}$ not topological, as we show in Fig. 3(b). Realizing the band inversion between the $\mathrm{U} 6 d$ and Sn $5 p$ bands at the $X$ point [see Fig. 3(c)] results in the fundamental gap $\Delta_{F}$ becoming topological. Additionally, we monitor the cancellation of the topological features inside the gaps $\Delta_{U}$ and $\Delta_{L}$. This cancellation is apparently due to a more complex overlap between various orbitals in 
the real calculation than the one assumed in the simplified model illustrated in Fig. 3(c), where one would, in principle, expect all three gaps to become topological $\left[Z_{2}=0\right.$ for the LHB and UHB, and $Z_{2}=1$ for the lowermost and topmost panels as shown in brackets in Fig. 3(c)].

To shed some additional light on the nature of the topological phase, we check the one-electron spectrum for a slab that is oriented along the 001 direction and terminated at the top by the U-Sn atomic plane and at the bottom by the Ni plane, where we expect to see the topological boundary states originating from the two surfaces around the TRIM points of the surface BZ where the band inversion occurs. We perform a real space transformation of our LDA + DMFT Hamiltonian that is possible due to the use of nonorthogonal tight-binding linear muffin-tin orbital representation seen as the unitary transformation of the linear muffin-tin orbital basis set [49]. The slab size containing 48 original unit cells along the $z$ axis provides a completely convergent surface spectrum. Our results are plotted in Fig. 4, where we show both the projected bulk spectrum [Fig. 4(a)] and the top and bottom surface states that are fattened according to the partial character of the topmost (U-Sn, green) and bottommost (Ni, red) atomic plane [Fig. 4(b)]. We find clear evidence of the Dirac-cone states that appear around the $\bar{\Gamma}$ point of the surface BZ [see the inset in Fig. 4(b)]. Note that, because we are considering the 001 surface, one bulk $X$ point is actually projected onto the surface $\bar{\Gamma}$ point; therefore, the appearance of the Dirac cone around the surface $\bar{\Gamma}$ point is likely due to the band inversion around the bulk $X$ point. We also identify Dirac cones in the immediate vicinity of the $\bar{X}$ point. However, we see that they do not span across the gap, likely due to hybridization with other surface states that appear in this energy range. Nevertheless, we can easily count that there is always an odd number of surface states that cross the Fermi level between the time reversal invariant momenta. For example, there are three "green" states and three "red" states that cross the Fermi energy shown in Fig. 4(b) between $\bar{\Gamma}$ and $\bar{X}$. The emergent physical picture resembles the case of $\mathrm{SmB}_{6}$ [11], where the band inversion between $\mathrm{Sm} 4 f$ and $5 d$ states around the bulk $X$ point and the apparent lack of trivial surface states for the 001 surface result in Dirac cones spanning across the energy gaps around the surface $\bar{X}$ and $\bar{\Gamma}$ points.

\section{B. Antiferromagnetic phase}

We now turn to discussing the results of our calculation for the low-temperature AFM phase of UNiSn. The origin of magnetism has been explained earlier [34] based on a molecular-field model, where, owing to the second-order effect in the magnetic exchange field, the $\Gamma_{3}$ doublet is split into two levels with a deduced magnetic moment value of approximately $2.6 \mu_{B}$. Here, our exact diagonalization for the $5 f$ states is almost identical to the static mean-field
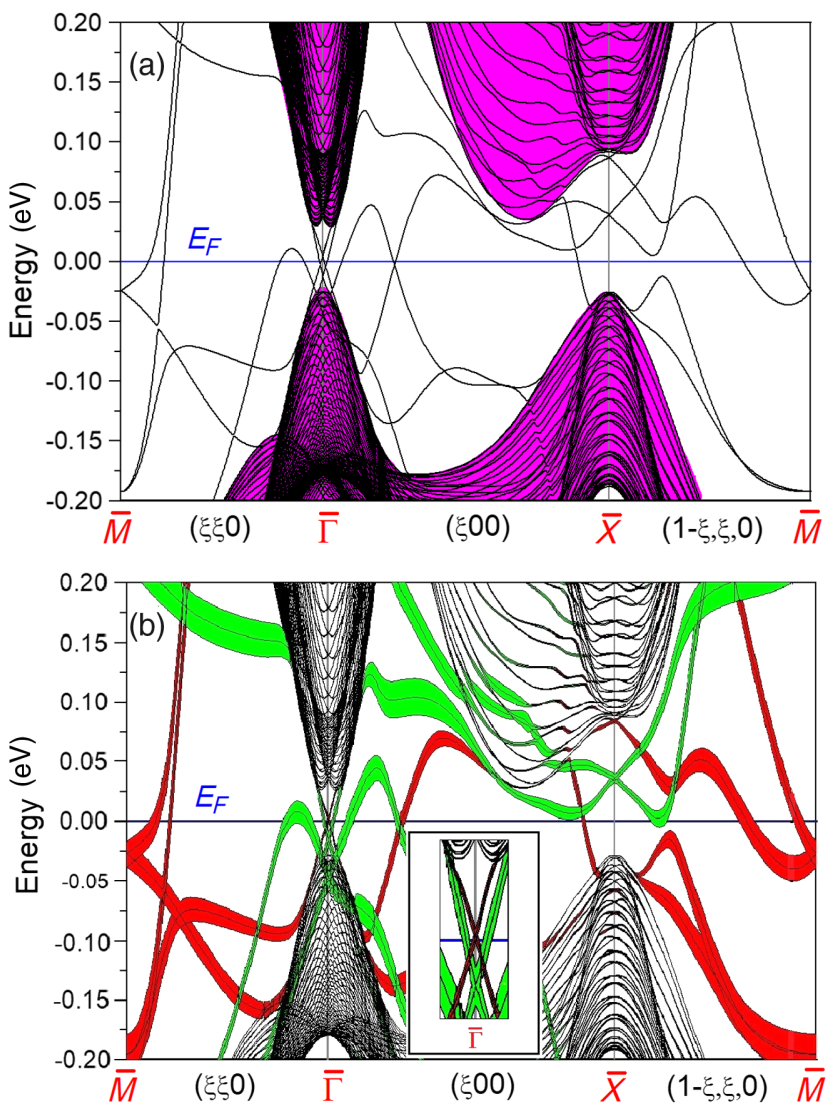

FIG. 4. (001) surface spectrum of UNiSn calculated along highsymmetry lines of the surface Brillouin zone, $\bar{\Gamma}(000), \bar{X}(100)$, and $\bar{M}\left(\frac{1}{2} \frac{1}{2} 0\right)$, using the LDA + DMFT method, assuming a $5 f^{2}$ $\Gamma_{3}$ doublet as a ground state. (a) The projected bulk spectrum is shown by magenta. (b) Top (green) and bottom (red) surface states are fattened according to the partial character of the topmost (U-Sn) and bottommost (Ni) atomic plane of the slab. The inset provides a close view of the Dirac states near the $\bar{\Gamma}$ point.

solution, because the double degeneracy of $\Gamma_{3}$ is broken and a single Slater determinant description suffices. It has been also proven earlier that the LDA + DMFT method reduces to the LDA $+U$ in the Hartree-Fock limit [50]. Our calculation with Slater integrals $F^{(0)}=0.15, F^{(2)}=0.3$, $F^{(4)}=0.2$, and $F^{(6)}=0.15$ in Rydberg units, converges to an antiferromagnetic state with a total magnetic moment of $2.1 \mu_{B}\left(+3.2 \mu_{B}\right.$ for its orbital and $-1.1 \mu_{B}$ for its spin counterparts), slightly larger than the experimentally deduced value of $1.55 \mu_{B}$ [32]. This result is in agreement with previous works $[36,37]$ that also point out the inclusion of spin fluctuations as a possible way to reduce these values. Our calculated spin density matrices resemble those obtained from the molecular-field exchange model [34].

Figure 5(a) shows our calculated band structure along the major high-symmetry directions of the BZ. A few energy bands are seen to cross the Fermi level, indicating the metallic nature of the solution. Since both time reversal and inversion symmetries are now broken, it is interesting to see 

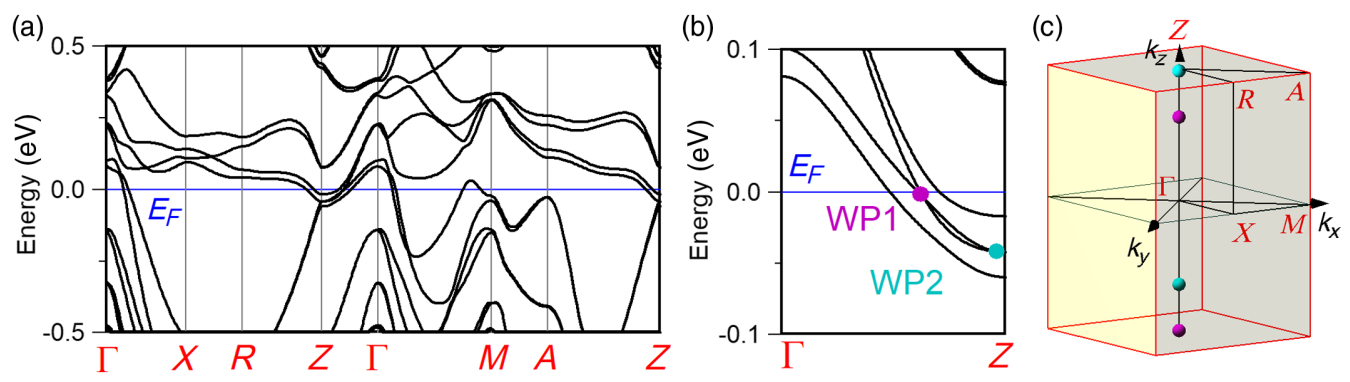

FIG. 5. Calculations for antiferromagnetic configuration of UNiSn. (a) Energy band dispersions along major high-symmetry lines of the Brillouin zone. (b) Enlarged area along the $\Gamma Z$ direction of the BZ showing the locations of the Weyl points with their coordinates $\mathbf{k}_{W P 1}=(0,0,0.317) 2 \pi / a$ (chiral positive, energy relative to $E_{F}$ is $-5 \mathrm{meV}$ ) and $\mathbf{k}_{W P 2}=(0,0,0.492) 2 \pi / a$ (chiral negative, energy relative to $E_{F}$ is $-42 \mathrm{meV}$ ). (c) Brillouin zone of the AFM UNiSn with the positions of the Weyl points (magenta refers to positive and cyan refers to negative chiral charges).

if there are any Weyl points in close proximity to the Fermi level. It is well known that the Weyl point acts as a Dirac monopole in $k$ space and produces a nonzero Berry flux through an area surrounding it [23,51]. We take advantage of this result and utilize a monopole mining method developed by us recently [25] in order to search for their locations (see the Appendix for details). This search is rewarded by finding two Weyl points that appear exactly along the $\Gamma Z$ line of the BZ, serving here as the magnetization direction. The corresponding band structure is shown in Fig. 5(b). Despite the Weyl points aligning with $\Gamma Z$, their positions along this line are found to be sensitive to the value of Hund's rule $J$ for $5 f$ electrons used in the calculation, as the latter controls the shape of the bands in this energy range. The WSM phase begins to appear starting with $J=0.2 \mathrm{eV}$ and persists for higher values of $J$. Figure 5(c) shows the positions of these Weyl points in the Brillouin zone for $J=0.3 \mathrm{eV}$. Their precise locations are given by the wave vectors $\mathbf{k}_{W P 1}=(0,0,0.317) 2 \pi / a$ (chiral positive, energy relative to $E_{F}$ is $-5 \mathrm{meV}$ ) and $\mathbf{k}_{W P 2}=(0,0,0.492) 2 \pi / a$ (chiral negative, energy relative to $E_{F}$ is $-42 \mathrm{meV}$ ). Unfortunately, in the absence of detailed knowledge about how the screening reduces the intra-atomic exchange interaction, these data can serve only as a guideline to possible experimental verification. Nevertheless, it should be easy to locate these Weyl points, since they are expected to appear along the $\Gamma Z$ line regardless of the inaccuracies in our predictions of their coordinates. Also, it would be interesting to check the value of the longitudinal magnetoresistance, whose negative sign could indicate that our predicted WSM phase and the associated chiral anomaly exist. Negative magnetoresistance is, in fact, reported for this compound [52,53], but the setup is related to measuring the (transverse) Hall coefficient.

To understand the physical origin behind a magnetizationinduced Weyl state in UNiSn, we introduce a $k \cdot p$ model for two relativistic orbitals with an inversion-breaking term.

The Hamiltonian reads

$$
H_{\mathrm{eff}}=\left(\begin{array}{cccc}
A(\mathbf{k})+\Delta_{1} & 0 & P k_{z}+i V k_{x} k_{y} & P k_{-}+V k_{z} k_{+} \\
0 & B(\mathbf{k})-\Delta_{1} & P k_{+}-V k_{z} k_{-} & -P k_{z}-i V k_{x} k_{y} \\
P k_{z}-i V k_{x} k_{y} & P k_{-}-V k_{z} k_{+} & C(\mathbf{k})+\Delta_{2} & 0 \\
P k_{+}+V k_{z} k_{-} & -P k_{z}+i V k_{x} k_{y} & 0 & D(\mathbf{k})-\Delta_{2}
\end{array}\right)
$$

where $k_{ \pm}=k_{x} \pm i k_{y}$ and diagonal elements are parametrized as follows: $A(\mathbf{k})=A_{0}+A_{1} \mathbf{k}^{2}, B(\mathbf{k})=B_{0}+$ $B_{1} \mathbf{k}^{2}-B_{2} \mathbf{k}^{4}, \quad C(\mathbf{k})=C_{0}+C_{1} \mathbf{k}^{2}-C_{2} \mathbf{k}^{4}$, and $D(\mathbf{k})=$ $D_{0}+D_{1} \mathbf{k}^{2}$ (we include quartic terms to allow multiple Weyl points to exist). The parameter $P$ controls the inversion breaking. A similar model was previously used to describe topological insulator and Weyl semimetal phases in zincblendelike structures [54]. Here, we apply a Zeeman splitting by setting the parameters $\Delta_{1,2} \neq 0$ along the magnetization $(z)$ axis. Once the effective "spin-up" and "spin-down" states cross, they produce Weyl points exactly along the 001 direction in the BZ, while the gap between these bands is open for all other $k$ points. We illustrate this behavior in Fig. 6(a), which shows the dispersion of the eigenvalues of Eq. (1) and the existence of two Weyl points along the $k_{z}$ axis. The parameters of the model are the following: $A_{0}=B_{0}=0.24, A_{1}=B_{1}=1, B_{2}=3.376, C_{0}=D_{0}=$ $-0.56, \quad C_{1}=D_{1}=0.3, \quad C_{2}=0.3, \quad P=0.9, \quad V=0.5$, $\Delta_{1}=0.3$, and $\Delta_{2}=0.6$. 

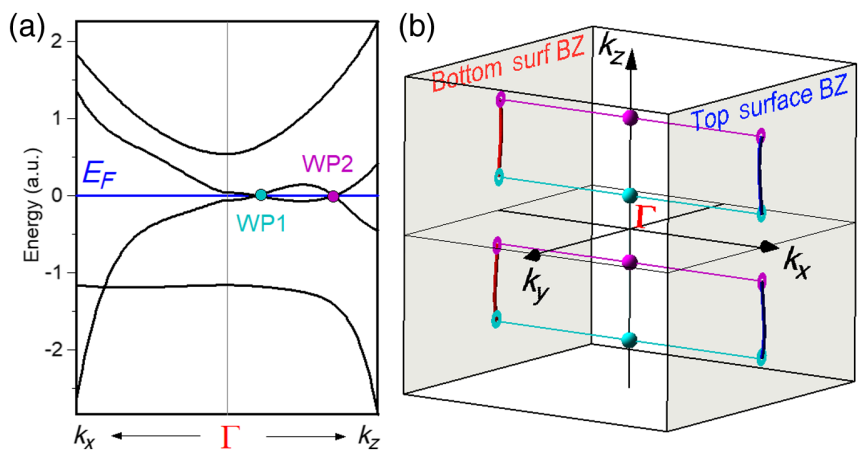

FIG. 6. (a) Dispersion of eigenstates of a $4 \times 4 k * p$ model used to illustrate the magnetization-induced Weyl semimetal state in UNiSn. The band structure is gapped for all $k$ points in the BZ except along the $\Gamma Z$ line, where the Weyl points are formed. (b) Position of the Weyl points in the $\mathrm{BZ}$ of the $k * p$ model periodized on the cubic lattice as well as shapes of the Fermi-arc surface states for the slab geometry with the normal along the $x$ axis. Magenta refers to positive and cyan refers to negative chiral charges.

One of the most striking features of Weyl semimetals is the presence of the Fermi arcs in their one-electron surface spectra [9]. Although computations of their shapes are possible via a self-consistent supercell (slab) calculation of the surface energy bands, given the variety of regular Fermi states that emerge from our AFM calculation together with the fact that the Weyl points are not exactly pinned at the Fermi level, it is hard to resolve them in the actual surface spectrum of UNiSn. Nevertheless, since the arcs connect the Weyl points of different chirality, one can expect the existence of long arclike features in UNiSn that should be protected from perturbations such as disorder [39].

To illustrate the shape of the Fermi arcs, we perform the diagonalization for the $k * p$ model that is periodized on the cubic lattice with subsequent construction of the Hamiltonian for the slab oriented perpendicular to the $x$ axis. The results are shown in Fig. 6(b), where almost straight Fermi arcs stretched along the $k_{z}$ axis are seen to connect the Weyl points of opposite chirality. The parameters of the model are exactly the same as used in Fig. 6(a). If these numbers are tweaked a little to simulate the case seen in Fig. 5(b) for UNiSn so that one Weyl point sinks just below the Fermi level producing a small Weyl Fermi sphere, the portion of the arc merging with this Weyl point rotates slightly away from the $k_{z}$ axis and now merges into the Fermi circle originating from the projection of the Weyl sphere to the surface BZ. At the end, we note that the limit of straight arc geometry was recently found [39] to be remarkably disorder tolerant, making it capable of producing ultrahigh conductivities of WSM nanostructures [40]. The present physical picture could, therefore, serve as one way to engineer such arcs and make a control of topological surface transport possible.

\section{CONCLUSION}

In conclusion, based on a computational approach combining the density functional theory of electronic structure and the dynamical mean-field theory of strong correlations, we show that two topological phases of quantum matter, topological insulator and Weyl semimetal, accompany the unconventional insulator-metal transition in the $5 f$ electron compound UNiSn. We uncover the physical origin of its topological insulator behavior via the occurrence of multiple band inversions between localized $f$ electrons and regular band states. We also conclude that the magnetic ordering triggers the Weyl state with the nodal points appearing along the magnetization direction.

Our study reveals interesting opportunities for finding other topological phase transitions in strongly correlated systems. Of particular interest are some noncentrosymmetric actinide compounds. A sister compound UPtSn is known to exhibit properties that are similar to UNiSn [33]. Another series that has been studied in the past through transport, heat capacity, neutron diffraction, and magnetic measurements is given by the uranium compounds in an expanded half-Heusler structure $\left[\mathrm{MgCu}_{4} \mathrm{Sn}\right.$ type, cF24-F43m, derived from the cubic $\mathrm{AuBe}_{5}$ lattice.] Among those, $\mathrm{UCu}_{4} \mathrm{Pd}$ is one of the most heavily investigated compounds owing to its strange dependence of the resistivity, magnetization, and specific heat, which scale as $T^{1 / 3}$ for temperatures below $10 \mathrm{~K}$ [55]. The quantum critical point and non-Fermi-liquid behavior of this compound is fairly well understood, but the transition between low- and higher-temperature phases has not fully been explained. Other famous systems to mention here are $\mathrm{UCu}_{4} \mathrm{Ni}$ [56] and $\mathrm{UPt}_{4} \mathrm{Au}$ [57]. Understanding the interplay between delocalized band electrons and correlated $5 f$ states known for their largest spin-orbit coupling is expected to provide an ideal playground for studying topological properties of interacting electrons.

\section{ACKNOWLEDGMENTS}

The work was supported by National Science Foundation DMR Grant No. 1832728. X. W. was supported by National Science Foundation of China, Grants No. 11525417 and No. 11834006.

\section{APPENDIX: THEORETICAL DETAILS}

Schematically, the LDA + DMFT method requires a self-consistent solution of the Dyson equation

$$
\left[\omega 1-H_{0}(\mathbf{k})-\Sigma(\omega)\right] G(\mathbf{k}, \omega)=1
$$

for the one-electron Green function $G(\mathbf{k}, \omega)$. The poles of its momentum-integrated function $G_{\mathrm{loc}}(\omega)$ contain information about the true local spectrum of excitations [31]. Here, $H_{0}(\mathbf{k})$ is the effective single-particle Hamiltonian, while $\Sigma(\omega)$ is a local self-energy operator. To improve the 
speed of the calculation, we earlier proposed a representation of the self-energy in terms of the Laurent series [45]

$$
\Sigma(\omega)=\Sigma(\infty)+\sum_{i} V_{i}^{+}\left(\omega-P_{i}\right)^{-1} V_{i},
$$

where weights $V_{i}^{+}$and $V_{i}$ and poles $P_{i}$ are generally matrices. Such a form of the self-energy allows us to replace the nonlinear (over energy) Dyson equation by a linear Schrödinger-like equation in extended subset of "pole states," which is clear due to a mathematical identity

$$
\begin{aligned}
& \left(\begin{array}{cc}
\omega-H_{0}(\mathbf{k})-\Sigma(\infty) & V^{+} \\
V & \omega-P
\end{array}\right)^{-1} \\
& \quad=\left(\begin{array}{cc}
{\left[\omega-H_{0}(\mathbf{k})-\Sigma(\infty)-V^{+}(\omega-P)^{-1} V\right]^{-1}} & \ldots \\
\vdots & \ddots
\end{array}\right),
\end{aligned}
$$

which relates our original matrix inversion required to find $G(\mathbf{k}, \omega)$ (first element in the matrix from the right) to the matrix inversion in the extended "pole space."

For the problem of UNiSn, we first exactly diagonalize the interacting Hamiltonian for the atomic $5 f$ electrons in the $f^{1}, f^{2}$, and $f^{3}$ configurations using the set of Slater integrals and the positions of the $f$ levels described in the main text. Second, the $f$-electron self-energy is extracted via the calculation of the atomic $5 f$ Green function describing the one-electron addition $\left(f^{2} \rightarrow f^{3}\right)$ and removal $\left(f^{2} \rightarrow f^{1}\right)$ processes to and from the $\Gamma_{3} f^{2}$ ground state. Third, the expansion (A2) for the self-energy is utilized, and the poles of the Green function matrix (A3) are found by diagonalization. Fourth, the Fermi level is adjusted, the charge density is calculated, and the entire procedure is made self-consistent. Finally, the poles of the self-consistent LDA + DMFT Green function (A3) are plotted in Figs. 2, 4, and 5.

To study topological invariants, we take advantage of a recent proof [46] that utilizing pole representation (A2) makes the topological indices $Z_{2}$ of the interacting system [right part of Eq. (A3)] and the noninteracting one [left part of Eq. (A3)] equivalent. The corresponding eigenstates of Eq. (A3) behave as effective quasiparticles described by the Bloch waves $|\mathbf{k} j\rangle$ and can be used for Berry phase calculations as follows. We represent the BZ by reciprocal lattice translations $\mathbf{G}_{\nu=1,2,3}$ and divide it onto $N_{1} \times N_{2} \times$ $N_{3}$ microcells. Each microcell is spanned by primitive vectors $\mathbf{q}_{\nu=1,2,3}=\mathbf{G}_{\nu} / N_{\nu}$ with its origin given by the grid of $\mathbf{k}$ points represented by three integers $n_{\nu}=0, N_{\nu}-1$ as $\mathbf{k}=n_{1} \mathbf{q}_{1}+n_{2} \mathbf{q}_{2}+n_{3} \mathbf{q}_{3}$. For each $\mathbf{k}$, we define a socalled link field that was introduced before [21] while evaluating the Berry phase using the finite difference method:

$$
U_{\mathbf{q}}(\mathbf{k})=\frac{\operatorname{det}\left[\left\langle\mathbf{k}+\mathbf{q} j^{\prime}\left|e^{i \mathbf{q r}}\right| \mathbf{k} j\right\rangle\right]}{\left|\operatorname{det}\left[\left\langle\mathbf{k}+\mathbf{q} j^{\prime}\left|e^{i \mathbf{q r}}\right| \mathbf{k} j\right\rangle\right]\right|}
$$

The Berry flux through each face of the microcell (plaquette) can be conveniently encoded into the following formula [21]:

$$
2 \pi \Phi \equiv \operatorname{Im} \ln \left[\frac{U_{\mathbf{q}_{\mu}}(\mathbf{k}) U_{\mathbf{q}_{\nu}}\left(\mathbf{k}+\mathbf{q}_{\mu}\right)}{U_{\mathbf{q}_{\nu}}(\mathbf{k}) U_{\mathbf{q}_{\mu}}\left(\mathbf{k}+\mathbf{q}_{\nu}\right)}\right] .
$$

For evaluating $Z_{2}$ invariants of the TI phase, we sum Berry fluxes through those plaquettes that constitute twodimensional tori introduced in Ref. [20] (pairs of reciprocal vectors $G_{\mu} G_{\nu}$ with an origin either at zero or shifted halfway along the third vector $G_{\xi}$ ). For locating the Weyl points of the WSM phase, we use the fact that they should behave as Dirac monopoles inside the microcells whose Berry fluxes give their chiralities [51,58]. Thus, we need to sum Berry fluxes through faces of the microcells, which makes the procedure very similar to the one employed while evaluating $Z_{2}$ invariants, since the $3 \mathrm{D} \mathbf{k}$ grid can be viewed as spanned by pairs of the reciprocal vectors $G_{\mu} G_{\nu}$ with a fixed fractional value along the third vector $G_{\xi}$ [25]. We need only to take care of the fact that the flux through each plaquette as given by Eq. (A5) produces right- (alternatively, left-) handed circulation of the Berry connection, but the inner (or outer) normal should be chosen consistently for the total flux through each surface of the microcell. The advantage of this logarithmic formulation is a guarantee that the total flux is always an integer (and so are $Z_{2}$ invariants or the monopole's chiral charges), since individual contributions (A5) from adjacent plaquettes cancel each other up to an addition of $2 \pi n$.

[1] For a review, see, e.g., Colossal Magnetoresistive Oxides (Gordon and Breach, Amsterdam, 2000).

[2] For a review, see, e.g., J. G. Bednorz and K. A. Muller, Perovskite-Type Oxides-The New Approach to High-Tc Superconductivity, Rev. Mod. Phys. 60, 585 (1988).

[3] For a review, see, e.g., G. R. Stewart, Superconductivity in Iron Compounds, Rev. Mod. Phys. 83, 1589 (2011).

[4] For a review, see, e.g., D. G. Koskimaki and K. A. Gschneidner, Jr., in Handbook on the Physics and Chemistry of Rare Earths, edited by K. A. Gschneidner, Jr. and L. R. Eyring (North-Holland, Amsterdam, 1978).

[5] For a review, see, e.g., A. Lawson, Plutonium Magic (Los Alamos Science, Los Alamos, 2006), p. 30.

[6] For a review, see, e.g., Q. Si and F. Steglich, Heavy Fermions and Quantum Phase Transitions, Science 329, 1161 (2010).

[7] For a review, see, e.g., M.Z. Hasan and C. L. Kane, Colloquium: Topological Insulators, Rev. Mod. Phys. 82, 3045 (2010). 
[8] For a review, see, e.g., N. P. Armitage, E. J. Mele, and A. Vishwanath, Weyl and Dirac Semimetals in ThreeDimensional Solids, Rev. Mod. Phys. 90, 015001 (2018).

[9] X. Wan, A. M. Turner, A. Vishwanath, and S. Y. Savrasov, Topological Semimetal and Fermi-Arc Surface States in the Electronic Structure of Pyrochlore Iridates, Phys. Rev. B 83, 205101 (2011).

[10] For a review, see, e.g., M. Dzero, J. Xia, V. Galitski, and P. Coleman, Topological Kondo Insulators, Annu. Rev. Condens. Matter Phys. 7, 249 (2016).

[11] F. Lu, J. Zhao, H. Weng, Z. Fang, and X. Dai, Correlated Topological Insulators with Mixed Valence, Phys. Rev. Lett. 110, 096401 (2013).

[12] B. Yan, L. Müchler, X.-L. Qi, S.-C. Zhang, and C. Felser, Topological Insulators in Filled Skutterudites, Phys. Rev. B 85, 165125 (2012).

[13] X. Zhang, H. Zhang, J. Wang, C. Felser, and S.-C. Zhang, Actinide Topological Insulator Materials with Strong Interaction, Science 335, 1464 (2012).

[14] H.-H. Lai, S. E. Grefe, S. Paschen, and Q. Si, Weyl-Kondo Semimetal in Heavy-Fermion Systems, Proc. Natl. Acad. Sci. U.S.A. 115, 93 (2018).

[15] For a review, see, e.g., M. Imada, A. Fujimori, and Y. Tokura, Metal-Insulator Transitions, Rev. Mod. Phys. 70, 1039 (1998).

[16] For a review, see, e.g., A. Georges, G. Kotliar, W. Krauth, and M. J. Rozenberg, Dynamical Mean-Field Theory of Strongly Correlated Fermion Systems and the Limit of Infinite Dimensions, Rev. Mod. Phys. 68, 13 (1996).

[17] For a review, see, e.g., Theory of the Inhomogeneous Electron Gas, edited by S. Lundqvist and S. H. March (Plenum, New York, 1983).

[18] L. Fu and C. L. Kane, Topological Insulators with Inversion Symmetry, Phys. Rev. B 76, 045302 (2007).

[19] C. L. Kane and E. J. Mele, $Z_{2}$ Topological Order and the Quantum Spin Hall Effect, Phys. Rev. Lett. 95, 226801 (2005).

[20] J. E. Moore and L. Balents, Topological Invariants of TimeReversal-Invariant Band Structures, Phys. Rev. B 75, 121306 (2007).

[21] T. Fukui and Y. Hatsugai, Quantum Spin Hall Effect in Three Dimensional Materials: Lattice Computation of $Z_{2}$ Topological Invariants and Its Application to Bi and Sb, J. Phys. Soc. Jpn. 76, 053702 (2007).

[22] M. G. Vergniory, L. Elcoro, C. Felser, B. A. Bernevig, and Z. Wang, A Complete Catalogue of High-Quality Topological Materials, Nature (London) 566, 480 (2019).

[23] T. Zhang, Y. Jiang, Z. Song, H. Huang, Y. He, Z. Fang, H. Weng, and C. Fang, Catalogue of Topological Electronic Materials, Nature (London) 566, 475 (2019).

[24] F. Tang, H.-C. Po, A. Vishwanath, and X. Wan, Towards Ideal Topological Materials: Comprehensive Database Searches Using Symmetry Indicators, Nature (London) 566, 486 (2019).

[25] V. Ivanov and S. Y. Savrasov, A Monopole Mining Method for High Throughput Screening for Weyl Semimetals, Phys. Rev. B 99, 125124 (2019).

[26] S.-S. Lee and S. Ryu, Many-Body Generalization of the $Z_{2}$ Topological Invariant for the Quantum Spin Hall Effect, Phys. Rev. Lett. 100, 186807 (2008).
[27] Z. Wang, X.-L. Qi, and S.-C. Zhang, Topological Order Parameters for Interacting Topological Insulators, Phys. Rev. Lett. 105, 256803 (2010).

[28] S. Raghu, X.-L. Qi, C. Honerkamp, and S.-C. Zhang, Topological Mott Insulators, Phys. Rev. Lett. 100, 156401 (2008).

[29] D. Pesin and L. Balents, Mott Physics and Band Topology in Materials with Strong Spin-Orbit Interaction, Nat. Phys. 6, 376 (2010).

[30] S.-L. Yu, X.C. Xie, and J.-X. Li, Mott Physics and Topological Phase Transition in Correlated Dirac Fermions, Phys. Rev. Lett. 107, 010401 (2011).

[31] For a review, see, e.g., G. Kotliar, S. Y. Savrasov, K. Haule, V. S. Oudovenko, O. Parcollet, and C. A. Marianetti, Rev. Mod. Phys. 78, 865 (2006).

[32] T. Akazawa, T. Suzuki, F. Nakamura, T. Fujita, T. Takabatake, and H. Fujii, Anomalous Magnetic Transition in UNiSn, J. Phys. Soc. Jpn. 65, 3661 (1996).

[33] For a review, see, e.g., P. S. Riseborough, Heavy Fermion Semiconductors, Adv. Phys. 49, 257 (2000).

[34] Y. Aoki, T. Suzuki, T. Fujita, H. Kawanaka, T. Takabatake, and H. Fujii, Specific heat and magnetic susceptibility of $U_{1-x} T h_{x} N i S n$, Phys. Rev. B 47, 15060 (1993).

[35] K. R. Lea, M. J. M. Leask, and W. P. Wolf, The Raising of Angular Momentum Degeneracy of f-Electron Terms by Cubic Crystal Fields, J. Phys. Chem. Solids 23, 1381 (1962).

[36] J.-S. Kang, J.-G. Park, K. A. McEwen, C. G. Olson, S. K. Kwon, and B. I. Min, Temperature-Dependent ValenceBand Photoemission Study of UNiSn, Phys. Rev. B 64, 085101 (2001).

[37] P. M. Oppeneer, A. N. Yaresko, A. Ya. Perlov, V. N. Antonov, and H. Eschrig, Theory of the Anomalous Magnetic Phase Transition in UNiSn, Phys. Rev. B 54, R3706 (1996).

[38] For a review, see, e.g., Strong Correlations in Electronic Structure Calculations, edited by V. I. Anisimov (Gordon and Breach, Amsterdam, 2000).

[39] G. Resta, S.-T. Pi, X. Wan, and S. Y. Savrasov, High Surface Conductivity of Fermi Arc Electrons in Weyl Semimetals, Phys. Rev. B 97, 085142 (2018).

[40] C. Zhang, Z. Ni, J. Zhang, X. Yuan, Y. Liu, Y. Zou, Z. Liao, Y. Du, A. Narayan, H. Zhang, T. Gu, X. Zhu, L. Pi, S. Sanvito, X. Han, J. Zou, Y. Shi, X. Wan, S. Savrasov, and F. Xiu, Ultra-High Conductivity in Weyl Semimetal NbAs Nanobelts, Nat. Mater. 18, 482 (2019).

[41] C. H. Li, O. M. J. van't Erve, J. T. Robinson, Y. Liu, L. Li, and B. T. Jonker, Electrical Detection of Charge-CurrentInduced Spin Polarization due to Spin-Momentum Locking in $\mathrm{Bi}_{2} \mathrm{Se}_{3}$, Nat. Nanotechnol. 9, 218 (2014).

[42] X. Peng, Y. Yang, R. R. P. Singh, S. Y. Savrasov, and D. Yu, Spin Generation Via Bulk Spin Current in Three Dimensional Topological Insulators, Nat. Commun. 7, 10878 (2016).

[43] For recent applications of SELDA, see, e.g., S. Y. Savrasov, G. Resta, and X. Wan, Local Self-Energies for $V$ and $P d$ Emergent from a Non-Local LDA + FLEX Implementation, Phys. Rev. B 97, 155128 (2018).

[44] For a review, see, e.g., E. Gull, A. J. Millis, A. I. Lichtenstein, A. N. Rubtsov, M. Troyer, and P. Werner, Continuous-time 
Monte Carlo methods for quantum impurity models, Rev. Mod. Phys. 83, 349 (2011).

[45] S. Y. Savrasov, K. Haule, and G. Kotliar, Many-Body Electronic Structure of Americum Metal, Phys. Rev. Lett. 96, 036404 (2006).

[46] L. Wang, H. Jiang, X. Dai, and X. C. Xie, Pole Expansion of Self-Energy and Interaction Effect for Topological Insulators, Phys. Rev. B 85, 235135 (2012).

[47] S. Savrasov, G. Kotliar, and E. Abrahams, Electronic Correlations in Metallic Plutonium within Dynamical Mean-Field Picture, Nature (London) 410, 793 (2001).

[48] X. Wan, J. Zhou, and J. Dong, The Electronic Structures and Magnetic Properties of Perovskite Ruthenates from Constrained Orbital-Hybridization Calculations, Europhys. Lett. 92, 57007 (2010).

[49] O. K. Andersen and O. Jepsen, Explicit, First-Principles Tight-Binding Theory, Phys. Rev. Lett. 53, 2571 (1984).

[50] I. Yang, S. Savrasov, and G. Kotliar, Importance of Correlation Effects on Magnetic Anisotropy in $\mathrm{Fe}$ and Ni, Phys. Rev. Lett. 87, 216405 (2001).

[51] G. E. Volovik, Zeros in the Fermion Spectrum in Superfluid Systems as Diabolical Points, Pis'ma Zh. Eksp. Teor. Fiz. 46 (1987) [JETP Lett. 46, 98 (1987)].

[52] T. T. M. Palstra, G. J. Nieuwenhuys, R. F. M. Vlastuin, J. van den Berg, J. A. Mydosh, and K. H. J. Buschow, Magnetic and Electrical Properties of Several Equiatomic
Ternary U-Compounds, J. Magn. Magn. Mater. 67, 331 (1987).

[53] J. Diehl, H. Fischer, R. Kohler, C. Geibel, F. Steglich, Y. Maeda, T. Takabatake, and H. Fujii, Hall Effect and Magnetoresistance in UNiSn, Physica (Amsterdam) 186B-188B, 708 (1993).

[54] Y. Du, E.-J. Kan, H. Xu, S. Y. Savrasov, and X. Wan, Turning Copper Metal into Weyl Semimetal, Phys. Rev. B 97, 245104 (2018).

[55] A. Otop, S. Sullow, E. W. Scheidt, and J. A. Mydosh, Critical Reexamination of Non-Fermi-Liquid Characteristics of Moderately Disordered $\mathrm{UCu}_{4} P d U C u 4 P d$, Phys. Rev. B 77, 045121 (2008).

[56] M. A. L. de la Torre, M. A. Arranz, M. Ellerby, and K. A. McEwen, The Kondo Contribution to the Electrical Resistivity in $U C u_{5-x} N i_{x}$ and the Non-Fermi Liquid Behaviour of $U C u_{4} N i$, J. Phys. Condens. Matter 15, 2599 (2003).

[57] S. G. Thomas, W. W. Kim, M. Lenkewitz, and G. R. Stewart, Formation of the Heavy Fermion Ground State in $U \mathrm{Pt}_{4} M_{1}, M=A u, A g, C$, Physica (Amsterdam) 199B, 88 (1994).

[58] Z. Fang, N. Nagaosa, K. S. Takahashi, A. Asamitsu, R. Mathieu, T. Ogasawara, H. Yamada, M. Kawasaki, Y. Tokura, and K. Terakura, The Anomalous Hall Effect and Magnetic Monopoles in Momentum Space, Science 302, 92 (2003). 